\title{
How common are motor problems in children with a developmental disorder: rule or exception?
}

Stefanie Pieters*, Master in Educational Sciences, PhD student, Department of Experimental-Clinical and Health Psychology, Ghent University

Karen De Block, Master in Psychology, Department of Experimental-Clinical and Health Psychology, Ghent University

Jan Scheiris, Master in Psychology, Psychologist-Director rehabilitation centre

Maria Eyssen, MD, Paediatrics and Rehabilitation medicine, Belgian Health Care Knowledge Centre

Annemie Desoete, PhD, Department of Experimental-Clinical and Health Psychology, Ghent University

Dirk Deboutte, PhD, Professor, Child and Adolescent Psychiatry, Antwerp University and Ghent University

Hilde Van Waelvelde, PhD, Rehabilitation Sciences and Physiotherapy, Ghent University

Herbert Roeyers, PhD, Professor, Department of Experimental-Clinical and Health Psychology, Ghent University

*Correspondence to Stefanie Pieters, Ghent University, Department of Experimental-Clinical and Health Psychology, Henri Dunantlaan 2, 9000 Ghent, Belgium, Tel. +32(0)9-264 8619 , Fax. +32(0)9-264 64 89, E-mail:

Stefanie.Pieters@UGent.be 


\section{Acknowledgements}

This project was executed under the responsibility of the Belgian Health Care Knowledge Centre (KCE). We wish to thank Sig and the participating centres for the data collection.

\section{Conflicts of Interest Statement}

There are no conflicts of interest.

\section{Short title}

Motor problems in Developmental Disorders

\section{Key words}

- Developmental Coordination Disorder

- Learning disabilities

- Developmental disorders

- Co-morbidity

- Gender

\section{Key messages}

- Co-morbidity of motor problems with pervasive developmental disorder, a hyperkinetic disorder and/or a speech, language or learning disability varied between one fourth and one third of the children in this clinical sample.

- Co-morbidity rates of motor problems in developmental and behavioural disorders are lower than previously reported

- Co-morbidity of motor problems with pervasive developmental disorder, a hyperkinetic disorder and/or a speech, language or learning disability shows different patterns in boys and girls

- It is recommended to asses motor skills in children with developmental disorders

Word count main text: 3167 


\section{Abstract}

Background: Few co-morbidity studies have been conducted since the Leeds Consensus Statement on Developmental Coordination Disorder (DCD) in 2006. In this Statement, international cut-offs and inclusion criteria were agreed and consequently the status of DCD changed. Furthermore, most existing comorbidity studies are small clinical studies, rather than epidemiological studies, resulting in a broad range of co-morbidity rates. DCD has a higher incidence for boys in comparison with girls, questions arise if this preponderance remains the same in combination with other developmental disorders. Therefore, in this study we aimed to determine co-morbidity and gender differences of motor problems in children with a pervasive developmental disorder, a hyperkinetic disorder and/or a speech, language or learning disability.

Methods: Profiles of 3608 children (mean age 9y $1 \mathrm{mo}$ ) referred to rehabilitation centres for behavioural, developmental and sensorineural disorders were studied.

Results: Motor problems were reported in one fifth of the total sample. Comorbidity of motor problems in specific disorders varied between almost one fourth to more than one third. The male/female ratio was significantly higher in children with motor problems and 2 or more other disorders compared to children with motor problems and less than 2 other disorders.

Conclusions: This study indicates that co-morbidity of motor problems with other clinical disorders is not exceptional and developmental deviance is seldom specific to one domain. However, current co-morbidity studies tend to overestimate the number of children with motor problems. In addition, there may be different patterns of symptoms between the genders. These findings stress the importance of assessing motor skills in children with various developmental disorders.

\section{Introduction}

Severe motor problems without a known medical condition that interfere with children's daily life are recognized as 'Developmental Coordination Disorder' (DCD) in the Diagnostic and Statistical Manual of Mental Disorders (DSM-IV-TR) (American Psychiatric Association [APA] 2000). In the World Health Organization International Statistical Classification of Diseases and Related Health Problems, $10^{\text {th }}$ Revision (ICD-10) (World Health Organization 1992), the label 'Specific Developmental Disorder of Motor Function' is used to refer to this disability. DCD appears in $1.7 \%$ of the school-aged children and has a gender ratio of 1.8:1 males to females (Lingam et al. 2009). Moreover, motor problems exist as a co-morbid disability in children with other developmental disorders (Emck et al. 2009). Diagnosing motor problems is very important since recent findings show the pervasive impact of motor problems on children's performance in daily life or at school (Wang et al. 2009). Furthermore, developmental disorders are relatively persistent and whereas isolated problems are more likely to show a relatively good outcome, co-morbid disabilities increase the risk of long-term difficulties (Hellgren et al. 1993, Moffitt 1990, Rasmussen and Gillberg 2000). In this article, we will focus on motor problems in children with pervasive developmental disorders (PDD), hyperkinetic disorders, speech 
and language disabilities and learning disabilities and we will evaluate its prevalence as a co-morbidity in boys and girls.

PDD is a group of disabilities characterized by qualitative impairments in social interaction and in communication and by stereotyped and repetitive motor mannerisms (American Psychiatric Association [APA] 2000). PDD has a prevalence of $0.6 \%$ (Fombonne 2005) with a male predominance of about 4 to 1 (Fombonne 2009). A recent study found that $79 \%$ of the children with PDD had definite movement impairments and 10\% had borderline problems (Green et al. 2009). The range of co-morbid motor problems in PDD in small clinical sample studies is large, from 50\% to $85 \%$ (Manjiviona and Prior 1995, Mayes and Calhoun 2003, Miyahara et al. 1997).

Hyperkinetic disorder is characterized by a pattern of inattention and/or hyperactivity and impulsivity (World Health Organization 1992). In a systematic review, the prevalence for ADHD/Hyperkinetic disorder was 5.29\% (Polanczyk et al. 2007). The ratio of boys to girls is between 3:1 and 9:1 (Swanson et al. 1998). Hyperkinetic disorder is a heterogeneous condition, with frequent co-morbid disabilities such as motor problems (Fliers et al. 2008). Approximately 30\% to $50 \%$ of children with hyperkinetic disorder show motor problems affecting equally boys and girls (Fliers et al. 2008, Gaub and Carlson 1997, Gillberg et al. 2004, Sergeant et al. 2006)

Children with speech and language disabilities fail to develop language at a normal rate without an explanation of a physical impairment or neurological disease (American Psychiatric Association [APA] 2000). They often have difficulties with tasks involving motor control in speaking. Speech and language disabilities occur in $6.3 \%$ of children with a male rate approximately double in comparison with females (Pinborough-Zimmerman et al. 2007). A recent study found that children with speech and language disabilities $(27.3 \%)$ had a higher chance than children without this disability $(11.4 \%)$ to have motor deficits (Cheng et al. 2009). There is a large range of reported co-morbid motor problems in speech and language disabilities going from $20 \%$ to $71 \%$ (Cheng et al. 2009).

There are two main learning disabilities, namely reading disabilities with an impairment in the development of reading and spelling skills and mathematical learning disabilities with an impairment in mathematical skills. Reading disabilities affect 1 to $33 \%$ of children, depending on the orthographic consistency of the language (Ziegler et al. 2010) with a male predominance between 1.74 and 2 (Liederman et al. 2005). To the best of our knowledge, there are no large comorbidity studies on motor problems in children with reading disabilities. Studies investigating motor problems in a small clinical sample revealed co-morbidity rates ranging from 33.2 to $87.5 \%$ (Kaplan et al. 1998, Miyahara et al. 1997). Mathematical learning disabilities frequently occur in children with a prevalence rate between 5.9 and 13.8\% (Barbaresi et al. 2005). Mathematical learning disabilities have an almost similar prevalence in boys and girls (Lewis et al. 1994). Sometimes boys are doing even slightly better (GrossTsur et al. 1996, von Aster 2000). We are not aware of a study that looked at co-morbidity of motor problems in children with mathematical learning disabilities (except the other way around).

In summary, the debate on the prevalence and co-morbidity remains unresolved. Most of the mentioned co-morbidity rates are derived from small clinical samples, rather than from epidemiological studies, resulting in a broad range of divergent co-morbidity rates. In this study we aim to determine the comorbidity of motor problems in a large sample, in accordance with the ICD-10 
(World Health Organization 1992) and the Leeds Consensus Statement (Sugden et al. 2006). In this Statement, international cut-offs and inclusion criteria were agreed for clinical and research purposes. Since then, the double diagnoses of PDD and DCD is encouraged when appropriate, which is in contrast with the DSM-IV-TR. Furthermore, a co-morbid diagnosis of mental disability and DCD is not longer advised, in the opposite of the ICD-10 and the DSM-IV-TR in which the motor problems must be in excess of those typically associated with a mental disability. The present study is one of the few studies on co-morbidity of motor problems with other disorders since the Leeds Consensus Statement on DCD in 2006. In addition, there is some controversy about co-morbidity patterns between the genders. Therefore, this study attempts to examine both gaps.

\section{Methods}

\section{Participants}

Records of the total yearly population of 31 rehabilitation centres, focusing mostly on ambulant rehabilitation of children and having a diagnostic and therapeutic task, were systematically collected and analyzed. Main target groups of these centres are PDD, hyperkinetic disorders, speech and language disabilities, learning disabilities, mental disabilities and conductive and sensorineural hearing loss. Centres are involved in the identification and the clarification of the nature, type and degree of the various developmental disorders. Outpatient therapy can be offered in various domains: language, speech and communication skills, (psycho) motor, (meta) cognitive, social-behavioural and emotional-affective abilities, 2 or 3 times a week. The team consists of different disciplines: physicians, social workers, psychologists, masters in educational sciences, speech therapists, audiologists, occupational therapists and physiotherapists. Rehabilitation involves at least three different team members with a different discipline providing face-toface treatment.

The sample comprised 3608 children, including all patients on a yearly basis whatever their main diagnosis. Children were between 1 and 21 years with a mean age of 9 years 1 month (SD 2y 6mo). Children between 5 and 12 years were the most common age group $(87 \%)$. The male/female ratio in the total sample was $2 / 1$. From these children, $81.4 \%$ attended regular education, $15.3 \%$ attended special education and $3.3 \%$ was not going to school.

\section{Design and procedure}

During 1 year and starting on January 1st, 2006, all Dutch-speaking rehabilitation centres in Belgium registered client information including gender, the main disability and (eventually) co-morbid disabilities based on the ICD-10 classification. Whereas the index disability was defined as the main disability or the most impairing disability in daily life, a co-morbid disability was defined as another important disability. Every child underwent a quite similar battery of standardized tests. Tests that were commonly used in the diagnostic protocol for DCD were the Movement Assessment Battery for Children (Henderson and Sugden 1992) and the Bruininks-Oseretsky Test of Motor Proficiency (Bruininks 1978). In order to register a main or a co-morbid disability, a score below percentile 10 on a standardized test and the presence of the defined clinical 
features by ICD-10 (the category 'Specific Developmental Disorder of Motor Function'), were required for a clinical diagnosis. Criteria described by the ICD10 are quite similar to those in the DSM-IV for DCD (Henderson and Barnett 1998). The centres received six-months training, a manual of the registration system and extra support if needed. ICD-10 classification codes were compared and discussed to develop a uniform application. Informed consents were obtained to use data for scientific purposes. When parents did not give permission, data were excluded. All processing of data was done anonymously. The study was in accordance with the privacy law (included in the agreement of the clients appealing to rehabilitation centres). All data were cleaned for data validation purposes. Data were reviewed and a post hoc random sample verification was performed (including 1\% of the data) to look for clerical errors. Researchers telephonically checked if the obtained data in the registration form were in correspondence with the clinical diagnosis in the centre. All telephonically received information was consistent with the ICD-codes (F80, F81, F84, F90 etc.) in the database.

\section{Analysis of data}

The participants were grouped with respect to presence of co-morbid motor problems. The number of children with motor problems and the gender ratio are presented in Table 1. Frequencies were calculated and Pearson's chisquare tests were conducted. Descriptive statistics compared the proportion of comorbid motor problems with the general population (1.7\%) (Lingam et al. 2009) using the test based on binomial distribution.

\section{Results}

The proportion of children with motor problems (registered with ICD-10 code $\mathrm{F} 82$ ) was $20.4 \%$ ( $\mathrm{n}=735$ out of the 3608 children). In most of these cases $(79.8 \%)$, it was registered as a co-morbid disability, in $5.3 \%$ it was the only diagnosis. All 735 children were at least of average intelligence (IQ>70). The male/female ratio was 2.6/1 and the age range was between 3 and 17 years (mean $9 y$ 1mo, SD $2 y 3 \mathrm{mo}$ ). From the children with motor problems, a minority had no other behavioural or developmental disorder. The majority had one (40.3\%), two $(43.7 \%)$, or three or more $(10.7 \%)$ behavioural and/or developmental disorders (see Table 1). In addition, the proportion of boys versus girls was significantly higher in children with motor problems and 2 or more other disorders compared to children with motor problems and less than 2 other disorders $\left(\chi^{2}=6,14 ; \mathrm{df}=1\right.$; $p<.01)$.

\section{Insert Table 1 about here}

In Table 2, an overview is outlined of the motor problems in the different behavioural and developmental disorders with disregard of a registration as a main or a co-morbid disability. For each diagnostic category (e.g. PDD), all children with this diagnosis either as a main or a co-morbid disability were taken into account. This means that one child can be counted more than once.

Binomial analysis revealed that there were significantly more co-morbid motor problems in speech and language disabilities or F80 (33.7\%) as opposed to $1.7 \%$ (Lingam et al. 2009) in the general population ( $p<.0001)$. Significantly more 
motor problems have also been established in learning disabilities or F81 (24.8\%) as opposed in the general population $(p<.0001)$. In addition, $25 \%$ of the children with PDD (or F84) and $23.9 \%$ of the children with a hyperkinetic disorder (or F90) had motor problems, which is significantly more in comparison with the general prevalence of motor problems (both $p<.0001$ ).

\section{Insert Table 2 about here}

When children with a 'Specific developmental disorder of scholastic skills' (F81) were studied more in detail, most children (63.1\%) had a 'Mixed disorder of scholastic skills' (this is a disability in reading or spelling together with a disability in mathematics). The other children had an isolated reading disability $(18.0 \%)$, an isolated mathematical learning disability $(13.3 \%)$ or an isolated spelling disability $(5.6 \%)$. The male/female ratio in the children with a reading disability or a spelling disability was $2.6 / 1$ and $1.1 / 1$ in the mathematical learning disability group. When co-morbid motor problems were taken into account in the latter group $(25.5 \%)$, the male predominance increased significantly to $1.8\left(\chi^{2}=3,68 ; \mathrm{df}=1 ; p<.05\right)$. For the children with a combined reading disability and mathematical learning disability, a gender difference of $1.5 / 1$ was found with a significant $\left(\chi^{2}=4,23 ; \mathrm{df}=1 ; p<.05\right)$ increase to $2.0 / 1$ when co-morbid motor problems were taken into account $(24.6 \%)$. Next to a mathematical learning disability and a combined reading disability and mathematical learning disability, there was also a significant difference in male/female ratio whether or not motor problems were reported for hyperkinetic disorders $\left(\chi^{2}=6,85 ; \mathrm{df}=1 ; p<.01\right)$, with an increase from $2.9 / 1$ to $4.2 / 1$. This was not found for the other developmental disorders, namely PDD and speech and language disabilities.

\section{Discussion}

The present study aimed to gather co-morbidity and gender data on motor problems in children with PDD, hyperkinetic disorders, speech and language disabilities and learning disabilities after the Leeds Consensus Statement (Sugden et al. 2006). To our knowledge, no data about the relationship between DCD and other developmental disorders is currently available since this Statement.

\section{Co-morbidity}

Our results illustrate that co-morbidity of motor problems in other disabilities is not exceptional since developmental deviance is seldom specific to one domain. The co-morbidity of motor disabilities with other developmental and behavioural disorders ranges from $23.9 \%$ to $33.7 \%$ with the highest co-morbidity in speech and language disabilities. Our results suggest that motor problems in these disabilities exist more frequently than can be expected by chance. It is questionable whether these motor problems should be considered as a co-morbid disability or as another phenomenon with a different aetiology, as proposed by different models of co-morbidity (Pennington 2006, Rhee et al. 2005, Willcutt et al. 2005). Although the term co-morbidity is used in this paper because of its common use in literature and in clinical practice, we agree with Kaplan and colleagues (Kaplan et al. 2006) that the term 'co-occurrence' is more appropriate to describe associations among developmental disorders. Co-morbidity might assume that the underlying pathophysiological origins of these disorders are 
independent and not causally related while co-occurrence can be used in cases with assumptions of a shared or completely unrelated aetiology (Kaplan et al. 2006). In this study, the overlap of motor problems with other developmental disorders could be the result of a shared aetiology.

Findings in our study could assume that co-morbidity studies in small clinical samples tend to overestimate the number of children with motor problems. A possible explanation could be the used definition of motor problems. Another possible explanation could be that the population in our study is indeed less (PDD, hyperkinetic disorder, learning disability) or more (speech and language disability) severely affected than in other co-morbidity studies, because our study population has not been recruited in the context of an experimental study. Indeed, in contrast with the 79\% of motor problems in PDD (Green et al. 2009), only 25\% of the children with PDD in our dataset had motor problems. The same was the case for hyperkinetic disorder. In our dataset only $23.9 \%$ of the children with hyperkinetic disorder had motor problems, whereas this was the case of 30 to $50 \%$ according to the literature (Fliers et al. 2008, Gillberg et al. 2004). In addition, findings in this study support $24.8 \%$ of motor problems in children with learning disabilities, which is much less than the co-morbidity range from $33.2 \%$ to $87.5 \%$ (Kaplan et al. 1998, Miyahara et al. 1997). However, in our dataset we found a slightly higher co-morbidity of motor problems in speech and language disabilities (33.7\%) compared to the $27.3 \%$ from Cheng and colleagues (2009). These findings suggest at first the need for more epidemiological research on prevalence and co-morbidity rates. How to explain these differences in prevalence and co-morbidity rates? The Leeds Consensus Statement suggests to exclude children with IQ below 70, but in most previous studies this exclusion criterion was already adapted. A possible explanation is a more stringent adaptation of the criterion requiring a significant interference with the activities of daily living at home and/or at school. The recent study of Lingam and colleagues (2009) is in line with this finding by reporting a lower prevalence of DCD $(1.7 \%)$ in comparison with the prevalence of $6 \%$ described by the American Psychiatric Association (1994). This last study was also based on the 'stringent' application of the 4 DCD criteria.

\section{Gender}

Our results regarding male/female ratios in the different developmental and behavioural disorders are in line with those reported in the literature (Fombonne 2009, GrossTsur et al. 1996, Lewis et al. 1994, Liederman et al. 2005, Pinborough-Zimmerman et al. 2007, Swanson et al. 1998).

In addition, we found a significant gender difference in children with motor problems regarding the number of reported co-morbid disabilities: the male/female ratio was higher in the sample of children with motor problems and two or more co-morbid disabilities, with a significant higher preponderance in boys, in comparison to the male/female ratio in the sample of children with motor problems and less than two co-morbid disabilities. To the best of our knowledge, this was not mentioned in literature before.

Finally, a significant difference was found in the male/female ratio whether or not motor problems were reported for mathematical learning disabilities, combined reading disabilities and mathematical learning disabilities and hyperkinetic disorders but not for the other developmental disorders, namely PDD and speech and language disabilities. These results might be explained by a different pattern of symptoms between genders. Boys with a mathematical 
learning disability, a combined reading disability and mathematical learning disability or a hyperkinetic disorder seem to have motor problems more often than girls. This suggest a greater vulnerability of boys to motor problems. However, this might also be an artefact based on selection and referral criteria. For example, girls are thought to be less disruptive and are therefore underidentified and undertreated for hyperkinetic disorder in comparison with boys. Additional studies seem indicated.

\section{Clinical implications}

Our results indicate that co-morbidity is not exceptional, stressing the importance of assessing motor skills in children with PDD, hyperkinetic disorders, speech and language disabilities and learning disabilities. The lack of correct and complete diagnoses may negatively influence the choice of appropriate care. Early assessment of motor problems might have a positive influence on the psychological development in individuals with PDD, hyperkinetic disorders, speech and language disabilities and/or learning disabilities. If the motor weaknesses are taken into account, different interventions and treatments may be considered to address the problems.

\section{Strengths and limitations}

This study adds data since the Leeds Consensus Statement on co-morbidity and gender data on motor problems in a large sample of children with PDD, hyperkinetic disorders, speech and language disabilities and learning disabilities. Records of 3608 children in ambulatory rehabilitation centres, specialized in behavioural and developmental disorders were retrospectively investigated. The results should therefore be interpreted with care since the data are based on individuals who have documented disabilities in a rehabilitation centre based on an ICD-10 registration. Because of the multidisciplinary character of these centres, rather higher co-morbidity rates might be expected and it cannot be an explanation for the finding that current co-morbidity studies tend to overestimate the number of children with motor problems. Recruiting in these centres gave us a more broaden view on co-morbidity by making it possible to compare between various developmental disorders.

\section{Future research}

The overlap of motor problems with other developmental disorders could be the result of an aberrant brain development. Future research is necessary to find the pathophysiological mechanisms underlying motor problems and other developmental disorders. A better understanding would have implications for diagnosis and treatment. Further research could also differentiate between various aspects of motor functioning, like fine motor skills, gross motor skills and balance.

\section{References}




\section{Tables}

Table 1: Children registered with ICD-10 code F82 (motor problems), either as a main or a co-morbid diagnosis: number of additional disabilities and gender

\begin{tabular}{lccc}
\hline \multirow{2}{*}{ Motor problems } & \multicolumn{2}{c}{ Prevalence } & Male/female ratio \\
& Boys & Girls & \\
\hline+ no other disabilities & 26 & 13 & $2 / 1$ \\
\hline+1 other disability & 202 & 94 & $2.1 / 1$ \\
\hline+2 other disabilities & 244 & 77 & $3.2 / 1$ \\
\hline+3 or more other disabilities & 61 & 18 & $3.4 / 1$ \\
\hline Total & 533 & 202 & $2.7 / 1$ \\
\hline
\end{tabular}

Table 2: Motor problems in the different behavioural and developmental disorders (one child could be counted more than once)

\begin{tabular}{|c|c|c|c|c|c|c|c|c|c|c|}
\hline \multirow[t]{2}{*}{ Disability } & \multicolumn{2}{|c|}{$\begin{array}{l}\text { Prevalence of the } \\
\text { disability }\end{array}$} & \multicolumn{2}{|c|}{$\begin{array}{l}\text { Prevalence } \\
\text { of the disability }\end{array}$} & \multirow{2}{*}{$\begin{array}{c}\text { Male/ } \\
\text { female ratio }\end{array}$} & \multicolumn{2}{|c|}{$\begin{array}{l}\text { Prevalence of motor } \\
\text { problems in this } \\
\text { disability }\end{array}$} & \multicolumn{2}{|c|}{$\begin{array}{l}\text { Prevalence of motor } \\
\text { problems in this } \\
\text { disability }\end{array}$} & \multirow{2}{*}{$\begin{array}{l}\text { Male/female } \\
\text { ratio when } \\
\text { motor problems }\end{array}$} \\
\hline & $\mathrm{n}$ & $\%$ & Boys & Girls & & $\mathrm{n}$ & $\%$ & Boys & Girls & \\
\hline $\begin{array}{l}\text { Specific developmental disorders of } \\
\text { speech and language (code F80) }\end{array}$ & 1278 & 35.4 & 859 & 419 & $2.1 / 1$ & 431 & 33.7 & 297 & 134 & $2.2 / 1$ \\
\hline $\begin{array}{l}\text { Specific developmental disorders of } \\
\text { scholastic skills (code F81) }\end{array}$ & 1319 & 36.6 & 823 & 496 & $1.7 / 1$ & 320 & 24.8 & 216 & 104 & $2.1 / 1$ \\
\hline $\begin{array}{l}\text { Pervasive developmental disorders (code } \\
\text { F84) }\end{array}$ & 660 & 18.3 & 546 & 114 & $4.8 / 1$ & 165 & 25.0 & 143 & 22 & $6.5 / 1$ \\
\hline Hyperkinetic disorders (code F90) & 949 & 26.3 & 705 & 244 & $2.9 / 1$ & 225 & 23.9 & 182 & 43 & $4.2 / 1$ \\
\hline
\end{tabular}


American Psychiatric Association (1994) Diagnostic and statistical manual of mental disorders (4th edition (DSM-IV)), American Psychiatric Association, Washington, DC.

American Psychiatric Association [Apa] (2000) Diagnostic and statistical manual of mental disorders (4th edition, text revision (DSM-IV-TR)), American Psychiatric Association, Washington, DC.

Barbaresi, W. J., Katusic, S. K., Colligan, R. C., Weaver, A. L. \& Jacobsen, S. J. (2005) Learning disorder: Incidence in a population-based birth cohort, 1976-82, Rochester, Minn. Ambulatory Pediatrics, 5, 281-289.

Bruininks, R. H. (1978) Bruininks-Oseretsky Test of Motor Proficiency, Circle Pines MN: American Guidance Service.

Cheng, H. C., Chen, H. Y., Tsai, C. L., Chen, Y. J. \& Cherng, R. J. (2009) Comorbidity of motor and language impairments in preschool children of Taiwan. Research in Developmental Disabilities, 30, 1054-1061.

Emck, C., Bosscher, R., Beek, P. \& Doreleijers, T. (2009) Gross motor performance and self-perceived motor competence in children with emotional, behavioural, and pervasive developmental disorders: a review. Developmental Medicine and Child Neurology, 51, 501-517.

Fliers, E., Rommelse, N., Vermeulen, S., Altink, M., Buschgens, C. J. M., Faraone, S. V., Sergeant, J. A., Franke, B. \& Buitelaar, J. K. (2008) Motor coordination problems in children and adolescents with ADHD rated by parents and teachers: effects of age and gender. Journal of Neural Transmission, 115, 211-220.

Fombonne, E. (2005) The changing epidemiology of autism. Journal of Applied Research in Intellectual Disabilities, 18, 281-294.

Fombonne, E. (2009) Epidemiology of Pervasive Developmental Disorders. Pediatric Research, 65, 591-598.

Gaub, M. \& Carlson, C. L. (1997) Gender differences in ADHD: A meta-analysis and critical review. Journal of the American Academy of Child and Adolescent Psychiatry, 36, 1036-1045.

Gillberg, C., Gillberg, I. C., Rasmussen, P., Kadesjo, B., Soderstrom, H., Rastam, M., Johnson, M., Rothenberger, A. \& Niklasson, L. (2004) Co-existing disorders in ADHD - implications for diagnosis and intervention. European Child and Adolescent Psychiatry, 13, I80-I92.

Green, D., Charman, T., Pickles, A., Chandler, S., Loucas, T., Simonoff, E. \& Baird, G. (2009) Impairment in movement skills of children with autistic spectrum disorders. Developmental Medicine and Child Neurology, 51, 311-316.

Grosstsur, V., Manor, O. \& Shalev, R. S. (1996) Developmental dyscalculia: Prevalence and demographic features. Developmental Medicine and Child Neurology, 38, 25-33.

Hellgren, L., Gillberg, C., Gillberg, I. C. \& Enerskog, I. (1993) Children With Deficits In Attention, Motor Control And Perception (Damp) Almost Grown Up - General Health At 16 Years. Developmental Medicine and Child Neurology, 35, 881-892.

Henderson, S. E. \& Barnett, A. L. (1998) The classification of specific motor coordination disorders in children: some problems to be solved (Reprinted 
from Perspectives on the classification of specific developmental disorders). Human Movement Science, 17, 449-469.

Henderson, S. E. \& Sugden, D. A. (1992) The Movement Assessment Battery for

Children, The Psychological Corporation, London.

Kaplan, B., Crawford, S., Cantell, M., Kooistra, L. \& Dewey, D. (2006)

Comorbidity, co-occurrence, continuum: what's in a name? Child: Care,

Health and Development, 32, 723-731.

Kaplan, B. J., Wilson, B. N., Dewey, D. \& Crawford, S. G. (1998) DCD may not be a discrete disorder. Human Movement Science, 17, 471-490.

Lewis, C., Hitch, G. J. \& Walker, P. (1994) The Prevalence Of Specific Arithmetic Difficulties And Specific Reading Difficulties In 9-Year-Old To 10-Year-Old Boys And Girls. Journal of Child Psychology and Psychiatry and Allied Disciplines, 35, 283-292.

Liederman, J., Kantrowitz, L. \& Flannery, K. (2005) Male vulnerability to reading disability is not likely to be a myth: A call for new data. Journal of Learning Disabilities, 38, 109-129.

Lingam, R., Hunt, L., Golding, J., Jongmans, M. \& Emond, A. (2009) Prevalence of Developmental Coordination Disorder Using the DSM-IV at 7 Years of Age: A UK Population-Based Study. Pediatrics, 123, E693-E700.

Manjiviona, J. \& Prior, M. (1995) Comparison Of Asperger Syndrome And HighFunctioning Autistic-Children On A Test Of Motor Impairment. Journal of Autism and Developmental Disorders, 25, 23-39.

Mayes, S. D. \& Calhoun, S. L. (2003) Ability profiles in children with autism Influence of age and IQ. Autism, 7, 65-80.

Miyahara, M., Tsujii, M., Hori, M., Nakanishi, K., Kageyama, H. \& Sugiyama, T. (1997) Brief report: Motor incoordination in children with Asperger syndrome and learning disabilities. Journal of Autism and Developmental Disorders, 27, 595-603.

Moffitt, T. E. (1990) Juvenile-Delinquency And Attention Deficit Disorder - Boys Developmental Trajectories From Age 3 To Age 15. Child Development, 61, 893-910.

Pennington, B. F. (2006) From single to multiple deficit models of developmental disorders. Cognition, 101, 385-413.

Pinborough-Zimmerman, J., Satterfield, R., Miller, J., Bilder, D., Hossain, S. \& Mcmahon, W. (2007) Communication disorders: Prevalence and comorbid intellectual disability, autism, and emotional/behavioral disorders. American Journal of Speech-Language Pathology, 16, 359-367.

Polanczyk, G., De Lima, M. S., Horta, B. L., Biederman, J. \& Rohde, L. A. (2007) The worldwide prevalence of ADHD: A systematic review and metaregression analysis. American Journal of Psychiatry, 164, 942-948.

Rasmussen, P. \& Gillberg, C. (2000) Natural outcome of ADHD with developmental coordination disorder at age 22 years: A controlled, longitudinal, community-based study. Journal of the American Academy of Child and Adolescent Psychiatry, 39, 1424-1431.

Rhee, S. H., Hewitt, J. K., Corley, R. P., Willcutt, E. G. \& Pennington, B. F. (2005) Testing hypotheses regarding the causes of comorbidity: Examining the underlying deficits of comorbid disorders. Journal of Abnormal Psychology, 114, 346-362.

Sergeant, J. A., Piek, J. P. \& Oosterlaan, J. (2006) ADHD and DCD: A relationship in need of research. Human Movement Science, 25, 76-89. 
Sugden, D., Chambers, M. \& Utley, A. (2006) Leeds Consensus Statement 2006: Developmental Coordination Disorder as a Specific Learning Difficulty., DCD-UK/Dyscovery Centre, Leeds.

Swanson, J. M., Sergeant, J. A., Taylor, E., Sonuga-Barke, E. J. S., Jensen, P. S. \& Cantwell, D. P. (1998) Attention-deficit hyperactivity disorder and hyperkinetic disorder. Lancet, 351, 429-433.

Von Aster, M. (2000) Developmental cognitive neuropsychology of number processing and calculation: varieties of developmental dyscalculia. European Child and Adolescent Psychiatry, 9, 41-57.

Wang, T. N., Tseng, M. H., Wilson, B. N. \& Hu, F. C. (2009) Functional performance of children with developmental coordination disorder at home and at school. Developmental Medicine and Child Neurology, 51, 817-825.

Willcutt, E. G., Pennington, B. F., Olson, R. K., Chhabildas, N. \& Hulslander, J. (2005) Neuropsychological analyses of comorbidity between reading disability and attention deficit hyperactivity disorder: In search of the common deficit. Developmental Neuropsychology, 27, 35-78.

World Health Organization (1992) The tenth revision of the international classification of diseases and related health problems (ICD-10), World Health Organization, Geneva.

Ziegler, J. C., Bertrand, D., Tóth, D., Csépe, V., Reis, A., Faísca, L., Saine, N., Lyytinen, H., Vaessen, A. \& Blomert, L. (2010) Orthographic Depth and Its Impact on Universal Predictors of Reading. Psychological Science, in press. 